\title{
Learning Joint Multilingual Sentence Representations with Neural Machine Translation
}

\author{
Holger Schwenk \\ Facebook \\ AI Research \\ schwenk@ fb.com
}

\author{
Matthijs Douze \\ Facebook \\ AI Research \\ matthijsefb.com
}

\begin{abstract}
In this paper, we use the framework of neural machine translation to learn joint sentence representations across six very different languages. Our aim is that a representation which is independent of the language, is likely to capture the underlying semantics. We define a new crosslingual similarity measure, compare up to $1.4 \mathrm{M}$ sentence representations and study the characteristics of close sentences. We provide experimental evidence that sentences that are close in embedding space are indeed semantically highly related, but often have quite different structure and syntax. These relations also hold when comparing sentences in different languages.
\end{abstract}

\section{Introduction}

It is today common practice to use distributed representations of words, often called word embeddings, in almost all NLP applications. It has been shown that syntactic and semantic relations can be captured in this embedding space, see for instance (Mikolov et al., 2013). To process sequences of words, ie. sentences or small paragraphs, these word embeddings need to be "combined" into a representation of the whole sequence. Common approaches include: simple techniques like bag-of-words or some type of pooling, eg. (Arora et al., 2017), recursive neural networks, eg. (Socher et al., 2011), recurrent neural networks, in particular LSTMs, eg. (Cho et al., 2014), convolutional neural networks, eg. (Collobert and Weston, 2008; Zhang et al., 2015) or hierarchical approaches, eg. (Zhao et al., 2015).

In some NLP applications, both the input and output are sentences. A very popular approach to handle such tasks is the so-called "encoder- decoder approach", also named "sequence-tosequence learning (seq2seq)". The main idea is to first encode the input sentence into an internal representation, and then to generate the output sentence from this representation. A very successful application of this paradigm is neural machine translation (NMT), see for instance (Kalchbrenner and Blunsom, 2013; Cho et al., 2014; Sutskever et al., 2014). Current best practice is to use recurrent neural networks for the encoder and decoder, but alternative architectures like convolutional networks have been also explored.

The performance of these vanilla seq2seq models substantially degrades with the sequence length since it is difficult to encode long sequences into a single, fixed-size representation. A plausible solution is the so-called attention mechanism (Bahdanau et al., 2015): where the generation of each target word is conditioned on a weighted subset of source words, instead of the full sentence. NMT has been also extended to handle several source and/or target languages at once, with the goal of achieving better translation quality than with separately trained NMT systems, in particular for under resourced languages, see for instance (Dong et al., 2015; Zoph and Knight, 2016; Luong et al., 2015a; Firat et al., 2016a).

In this work, we aim at learning multilingual sentence representations, i.e. which are independent of the language. Since we have to compare these representations among each other, for the same or between multiple languages, we only consider representations of fixed size.

There are many motivations to learn such a multilingual sentence representation, in particular:

- it is likely to capture the underlying semantics of the sentence (since the meaning is the only common characteristic of a sentence formulated in several languages);

- it has the potential to transfer many sentence 
processing applications to other languages (classification, sentiment analysis, semantic similarity, etc), without the need for language specific training data;

- it enables multilingual search;

- such representation could be considered as sort of a continuous space interlingua.

To train these multilingual sentence embeddings we are using the framework of NMT with multiple encoders and decoders. We first describe our model in detail, relate it to existing research, and then present an experimental evaluation.

\section{Architecture}

We propose to use multiple encoders and decoders, one for each source and target language respectively. The notion of multiple input languages can be also extended to different modalities, e.g. speech and images. One can also envision to add classification tasks, in addition to sequence generation. Our ultimate goal is to jointly train this generic architecture on many tasks at once, to obtain a universal multilingual and -modal representation (see illustration in Figure 1). To ease the comparison and search, we are focusing on representations of fixed-size, independently of the length of the input (and output) sequence. This choice has certainly an impact on the performance for very long sequences, ie. in the order of more than fifty words, but we argue that such long sentences are probably not very frequent in every day communication. We would also like to emphasize that the goal of this work is not to improve NMT (for multiple languages), but to use the NMT framework to learn multilingual sentence embeddings. Once the system is trained, the decoders

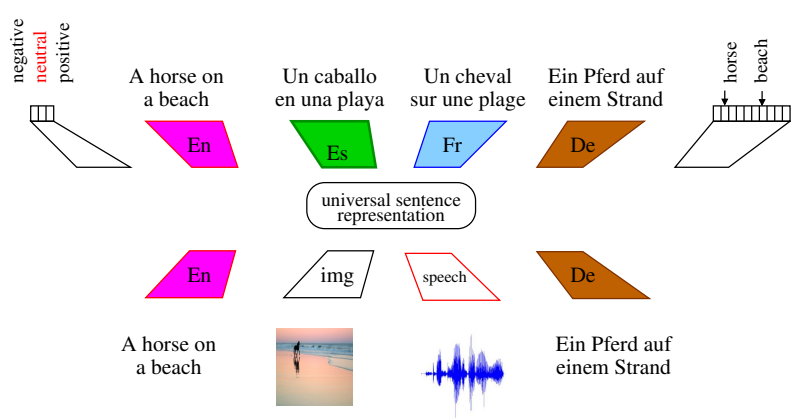

Figure 1: Generic multilingual and -modal encoder/decoder architecture. are not used any more. This means in particular that the usual attention mechanism cannot be used since the attention weights are usually conditioned on the decoder outputs. A possible solution could be to condition the attention on the inputs only, for instance so-called self-attention (Liu et al., 2016) or inner-attention (Lin et al., 2017).

To fix ideas, let us consider that we have corpora in $L$ different languages which can be pairwise or $N$-way parallel, $N \leq L$. This means that our architecture is composed of $L$ encoders and $L$ decoders respectively. However, this does not mean that we always provide input to all encoders, or targets for all decoders, but we change the used models at each mini-batch. One could for instance perform one mini-batch with two input languages and one output language (which requires an 3-way parallel corpus), and use one (different) input and output language in the next mini-batch (which require a bitext). We call this partial training paths. Note that we can also use monolingual data in this framework, ie. the input and output language is identical.

There are many possibilities to define partial training paths, with $1<M, N \leq L$.

1:1 translating from one source into one target language respectively.

M:1 presenting simultaneously several source languages at the input.

1:N translating from one source language into multiple target languages.

$\mathbf{M}: \mathbf{N}$ this is a combination of the preceding two strategies and the most general approach. Remember that not all inputs and outputs need to be present at each training step.

Our goal is to learn joint sentence representations, which are as close as possible when sentences are presented in different languages at the input. If we use 1:1 training, changing the language pair at each mini-batch (input and output), it is quite unlikely that the system would learn a common joint representation which is independent of the source language. A variant of 1:1 training is to always use the same decoder, but many different encoders. Since the decoder is shared for all the input languages, and the capacity of the model is limited, there's an incentive for the system to use the same representations for all the encoders. 

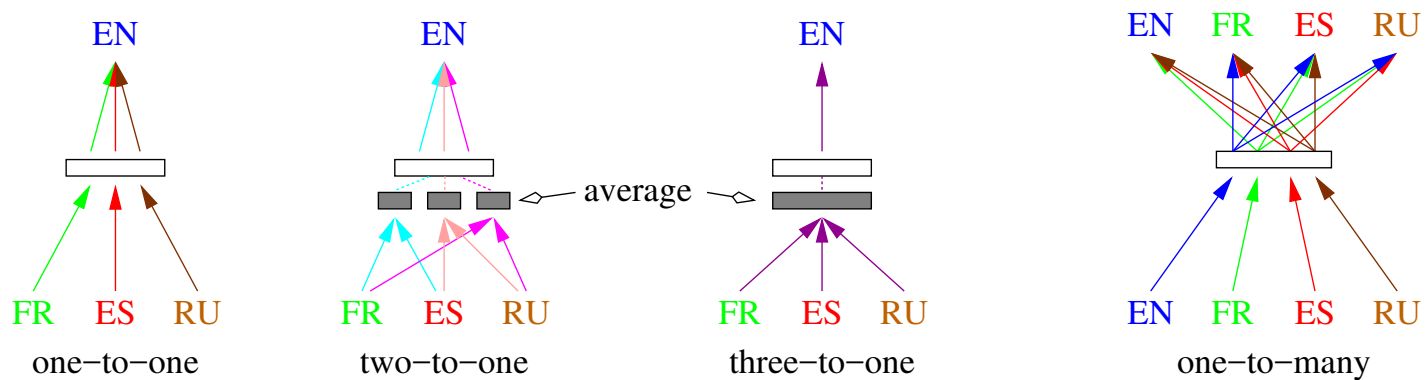

Figure 2: Possible partial training paths when four languages are available (En, Fr, Es and Ru). From left: 1:1, 2:1 and 3:1 strategy, using En as common target language.

Right: 1:3 strategy, translating from one source to the three other target languages.

This training strategy only requires bitexts with one common language (usually English). An important drawback, however, is that we will not obtain an embedding of this common language since it is never used at the input. ${ }^{1}$

Using multiple languages at the input at the same time and combining the corresponding sentence embeddings, ie. the M:1 strategy, has in principle the potential to learn joint sentence embeddings, if an appropriate technique is used to combine the individual embeddings. The most straightforward approach is to average the embeddings. This was used for instance in (Firat et al., 2016b) in a multilingual NMT system with attention. The joint embedding could be also enforced by some type of regularizer. Again, having one dedicated output language makes it impossible to learn a representation for it.

The 1:N strategy is an interesting extension of $1: 1$. The idea is translate from one input language simultaneously to all $L-1$ other languages, excluding the one at the input (ie. no autoencoder). The source and the set of target languages is changed at each mini-batch. By these means, every input language has at least one target language in common with all input languages, and each target language has at least one input language in common. On one hand hand, this strategy makes it possible to learn sentence embeddings for all languages, but one the other hand, it requires $L$-way parallel training data. Although bitexts are usually used in MT, there are also several corpora which can be aligned for more than two languages (eg. Eurpoarl, TED, UN). Finally, the N:M strat-

\footnotetext{
${ }^{1}$ One could also use the common output language at the input. This corresponds to training an auto-encoder which is easier than a translation model and may have an negative impact.
}

egy is the most generic one which combines all above techniques. These different training strategies are illustrated in Figure 2 for four languages.

\subsection{Related work}

The use of multiple encoders and decoders was first studied in the context of neural MT. Dong et al. (2015) used multiple decoders, i.e. 1:N training, to achieve improved NMT performance. Zoph and Knight (2016) and Firat et al. (2016b), on the other hand, used multiple encoders, i.e. M:1 training. It's not surprising that this complementarity improves MT quality, in comparison to one input language only. Many different configurations were explored by (Luong et al., 2015a) for seq2seq models. Firat et al. (2016a) were the first to use multiple encoders and decoders with a shared attention mechanism. This approach was further refined to enable zero-resource NMT (Firat et al., 2016b). Alternatively, it was proposed to handle multiple source and target languages with one encoder and decoder only, using a special token to indicate the target language (Johnson et al., 2016) to enable zero-shot NMT. To best of our knowledge, all these works focus on the improvement and extensions of seq2seq modeling, and fixed-sized vector representations have not analyzed in depth in a multilingual context.

Several publications consider joint representations in a multimodal context, usually text and images, for instance (Frome et al., 2013; Ngiam et al., 2011; Nakayama and Nishida, 2016). The usual approach is to optimize a distance or correlation between the two representations or predictive auto-encoders (Chandar et al., 2013). The same approach was applied to transliteration and captioning (Saha et al., 2016).

There is a large body of research on sentence 
representations. Common approaches include: simple techniques like bag-of-words or some type of pooling, eg (Arora et al., 2017), recursive NNs, eg. (Socher et al., 2011), recurrent NNs, in particular LSTMs, eg. (Cho et al., 2014), convolutional NNs, eg. (Collobert and Weston, 2008; Zhang et al., 2015) or hierarchical approaches, eg. (Zhao et al., 2015). In all these works, the sentence representations are learned for one language only. It is important to note that our multiple encoder/decoder architecture and the different training paths make no assumption on the type of encoder and decoder used. In principle, all these sentence representations methods could be used. This is left for future research.

There are several works on learning multilingual representations at document level (Hermann and Blunsom, 2014; Zhou et al., 2016b; Pham et al., 2015). (Hermann and Blunsom, 2014) proposed a compositional vector model to learn document level representations. Their model is based on bag of words/bi-gram composition. (Pham et al., 2015) directly learn a vector representations for sentences in the absence of compositional property. (Zhou et al., 2016b) learn bilingual document representation by minimizing Euclidean distance between document representations and their translation.

Other multilingual sentence representation learning techniques include BAE (Chandar et al., 2013) which trains bilingual autoencoders with the objective of minimizing reconstruction error between two languages, and BRAVE (Bilingual paRAgraph VEctors) (Mogadala and Rettinger, 2016) which learns both bilingual word embeddings and sentence embeddings from either sentence-aligned parallel corpora (BRAVE-S), or label-aligned non-parallel corpora (BRAVE-D).

Finally, many papers address the problem of learning bi- or multilingual word representations which are used to perform cross-lingual document classification. They are trained either on word alignments or sentence-aligned parallel corpora, or both. I-Matrix (Klementiev et al., 2012) uses word alignments to do multi-task learning, where each word is a single task and the objective is to move frequently aligned words closer in the joint embeddings space. DWA (Distributed Word Alignment) (Kociský et al., 2014) learns word alignments and bilingual word embeddings simultaneously using translation probability as objec- tive. Without using word alignments, BilBOWA (Gouews et al., 2014) optimizes both monolingual and bilingual objectives, uses Skip-gram as monolingual loss, while formulating the bilingual loss as Euclidean distance between bag-ofwords representations of aligned sentences. UnsupAlign (Luong et al., 2015b) learns bilingual word embeddings by extending the monolingual Skip-gram model with bilingual contexts based on word alignments within the sentence. TransGram (Coulmance et al., 2015) is similar to (Pham et al., 2015) but treats all words in the parallel sentence as context words, thus eliminating the need for word alignments.

\section{Evaluation protocol}

An important question is how to evaluate multilingual joint sentence embeddings. Let us first define some desired properties of such embeddings:

- multilingual closeness: the representations of the same sentence for different languages should be as similar as possible;

- semantic closeness: similar sentences should be also close in the embeddings space, ie. sentences conveying the same meaning, but not necessarily the syntactic structure and word choice;

- preservation of content: sentence representations are usually used in the context of a task, eg. classification, multilingual NMT or semantic relatedness. This requires that enough information is preserved in the representations to perform the task;

- scalability to many languages: it is desirable that the metric can be extended to many languages without important computational cost or need for human labeling of data.

Two main approaches have been used in the literature to evaluate multilingual sentence embeddings: 1) cross-lingual document classification based on the Reuters corpus, first described in (Klementiev et al., 2012); and 2) cross-lingual evaluation of semantic textual similarity (in short STS). This task was first introduced in the 2016 edition of SemEval (Agirre et al., 2016). Both tasks focus on the evaluation of joint sentence representations of two languages only. In the Reuters task, a document classifier is trained on English 
sentence representations and then applied to texts in another language, and in the opposite direction respectively. STS seeks to measure the degree of semantic equivalence between two sentences (or small paragraphs). Semantic similarity is expressed by a score between 0 (the two sentences are completely dissimilar) and 5 (the two sentences are completely equivalent). In 2016, a cross lingual task was introduced (Es/En) and extended to two more language pairs in 2017 ( $\mathrm{Ar} / \mathrm{En}$ and $\mathrm{Tr} / \mathrm{En})$.

In this work, we propose an additional evaluation framework for multilingual joint representations, based on similarity search. Our metric can be automatically calculated without the need of new human-labeled data and scaled to many languages and large corpora. We only need collections of $S$ sentences, and their translations in $L$ different languages, ie. $s_{i}^{p}, i=1 \ldots S, p=$ $1 \ldots L$. Such L-way parallel corpora are freely available, for instance Europarl ${ }^{2}$ (20 languages), the UN corpus, 6 languages (Ziemski et al., 2016), or TED, 23 languages, (Cettolo et al., 2012).

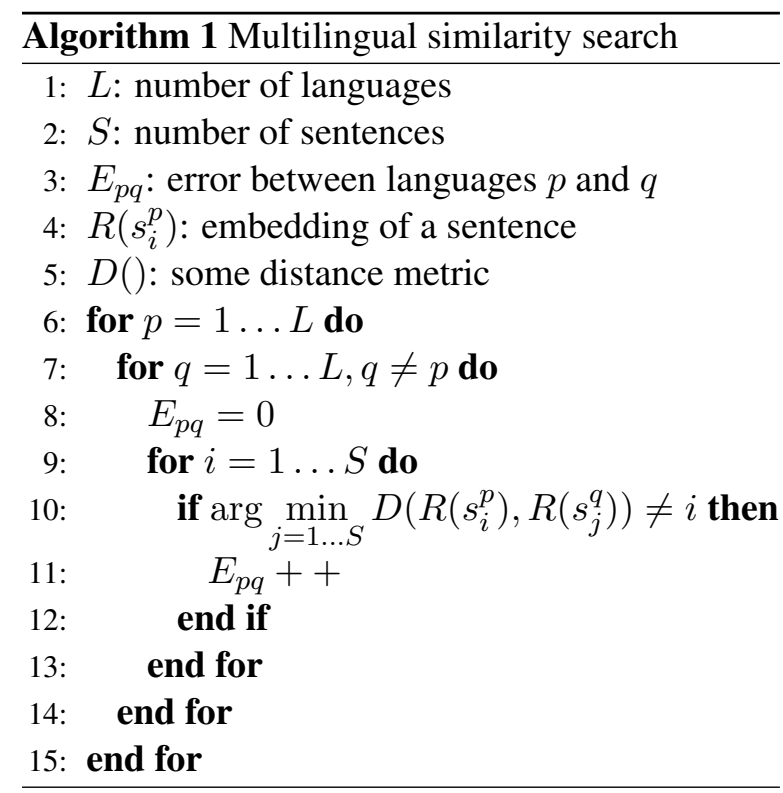

The details of our approach are given in algorithm 1 . The basic idea is to search the closest sentence in all $S$ sentences, and count an error if it is not the reference translation. This requires the calculation of $S^{2}$ distance metrics and makes only sense when there are no duplicate sentences in the corpus. With increasing $S$ it may be also likely that the corpus contains several alternative valid translations which could be closer than the

\footnotetext{
${ }^{2}$ http://www. statmt.org/europarl/
}

reference one. This is difficult to handle automatically at large scale and counted as error by our algorithm.

Similarity search mainly evaluates the multilingual closeness property and can be easily scaled to many languages. We will report results how the similarity error rate is influenced by the number of language pairs and the size of the corpus. We have compared three distance metrics: L2, inner product and cosine. In general, cosine performed best. Note that all metrics are equivalent if the vectors are normalized.

\section{Experimental evaluation}

We have performed all our experiments with the freely available UN corpus. It contains about $12 \mathrm{M}$ sentences in six languages (En, Fr, Es, Ru, Ar and $\mathrm{Zh}$ ). We have used the version which is 6-way parallel (about 8.3M sentences). This corpus comes with a predefined Dev and Test set (4000 sentences each). We lowercase all texts, limit the length of the training data to 50 words and use byte-pair encoding (BPE) with a 20k vocabulary. BPE allows to limit the size of the decoder output vocabulary, it has only a small impact on the sentence length $(\approx+20 \%)$ and it showed similar or even superior performance in NMT in comparison to many other techniques to limit the size of the output vocabulary (Sennrich et al., 2016). We have also found that BPE is very robust to spelling errors which is important when handling informal texts.

\subsection{Different network architectures}

In this work we only consider stacked LSTMs as encoders and decoders. In the vanilla seq2seq NMT model, the last state of the LSTM is used as sentence representation. There is also evidence that deeper architectures perform better in NMT than shallow ones, eg. (Zhou et al., 2016a; Wu et al., 2016). Following this tendency, we performed the first set of experiments with stacked LSTMs with three 512-dimensional hidden layers. Deeper architectures did not improve the performance.

We then switched to using BLSTMs followed by max-pooling (element-wise over the sequence length). We are not aware of works which use max-pooling in an NMT framework. One is indeed tempted to assume that max-pooling makes it more difficult to create a target sentence which preserves all information from the source sentence. On the other hand, max-pooling is success- 


\begin{tabular}{|c|c|c|c|c|}
\hline \multirow{2}{*}{$\begin{array}{l}\text { System } \\
\text { \#pairs: }\end{array}$} & \multicolumn{4}{|c|}{ Average Similarity Error } \\
\hline & $\begin{array}{c}\text { efs } \\
6\end{array}$ & $\begin{array}{c}\text { efsr } \\
10\end{array}$ & $\begin{array}{c}\text { efsra } \\
15\end{array}$ & $\begin{array}{c}\text { efsraz } \\
21\end{array}$ \\
\hline \multicolumn{5}{|c|}{ One-to-one systems: } \\
\hline efs-r & $1.97 \%$ & - & - & - \\
\hline efs-a & $2.09 \%$ & - & - & - \\
\hline efsr-a & $1.90 \%$ & $2.40 \%$ & - & - \\
\hline efsra-z & $1.91 \%$ & $2.26 \%$ & $2.51 \%$ & - \\
\hline \multicolumn{5}{|c|}{ One-to-many systems: } \\
\hline efsraz-all & $1.70 \%$ & $1.97 \%$ & $2.38 \%$ & $2.59 \%$ \\
\hline \multicolumn{5}{|c|}{ One-to-many systems, nhid=1024: } \\
\hline efsraz-all & $1.36 \%$ & $1.64 \%$ & $1.89 \%$ & $1.95 \%$ \\
\hline
\end{tabular}

Three layer LSTM, nhid=512 Sentence representation: last LSTM state

\begin{tabular}{|l||c|c|c|c|}
\hline \multicolumn{1}{|c||}{ System } & \multicolumn{4}{c|}{ Average Similarity Error } \\
\#pairs: & 6 & 10 & 15 & 21 \\
\hline \hline \multicolumn{1}{|l|}{ One-to-one systems: } \\
efs-r & $1.11 \%$ & - & - & - \\
efs-a & $1.03 \%$ & - & - & - \\
efsr-a & $1.11 \%$ & $1.31 \%$ & - & - \\
efsra-z & $1.01 \%$ & $1.19 \%$ & $1.25 \%$ & - \\
\hline
\end{tabular}

One-to-many systems:

\begin{tabular}{|l||l|l|l|l|} 
efsraz-all & $0.92 \%$ & $1.07 \%$ & $1.15 \%$ & $\mathbf{1 . 2 0 \%}$ \\
\hline
\end{tabular}

Table 1: Error rates of similarity search on the UN Dev corpus. Languages are abbreviated with the following letters: $\mathrm{e}=$ English, $\mathrm{f}=$ French, $\mathrm{s}=$ Spanish, $\mathrm{r}=$ Russian, $\mathrm{a}=$ Arabic, $\mathrm{z}=$ Chinese.

fully used in various sentence classification tasks, eg. (Conneau et al., 2017). It should be noted that the final sentence representation has twice the dimension of the BLSTM hidden layer.

The word embeddings are of size 384 for all models. We use vertical dropout with a value of 0.2 and gradients are clipped at 2. The initial learning rate is set to 0.01 and decreased each time performance on the Dev data does not improve. Performance is measured by perplexity for the decoders and similarity error at the embedding layer for the encoders. It is important to note that the similarity error rate can be only calculated once the whole development set is processed. Therefore it is not used to provide gradients to the encoders. Training is performed for up to five epochs with a batch size of 96 . For the smallest models, one iteration through the training data takes about $11 \mathrm{~h}$. Most models converge after two to three epochs.

Table 1 summarizes our results on the UN Dev corpus for several systems using the one-to-one and one-to-many partial training paths. We compare training of joint representations for three to six languages using LSTM or BLSTM encoders. In each column, we give the average similarity error over all $n(n+1) / 2$ language pairs. As an example, the system trained with En, Fr, Es and Ru at the input and Ar at the output ("efsr-a" in the third line), achieves an average similarity error of $1.90 \%$ over 6 language pairs ${ }^{3}$, column "efs", and $2.40 \%$ over 10 languages pairs ${ }^{4}$, column "efsr".

\footnotetext{
${ }^{3}$ En-Es, En-Fr, Es-En, Es-Fr, Fr-En and Fr-Es.

${ }^{4}$ En-Es, En-Fr, En-Ru, Es-En, Es-Fr, Es-Ru, Fr-En, Fr-Es,
}

We can make the following observations. First, using an BLSTM with max-pooling (Table 1 right) performs much better than an LSTM and using the last hidden state as sentence representation (Table 1 left). This was also observed for many monolingual tasks, eg. (Conneau et al., 2017). This is particularly true when the number of languages is increased. This performance gain does not result from the increased dimension of the sentence representation $(2 \times$ nhid $)$ since an 1024-dimensional LSTM only achieves $1.36 \%$ (see last line in Table 1 left). Second, increasing the number of languages for which we seek a joint sentence embedding does not seem to make the task harder: the system trained on all languages achieves the same results $(1.01 \%)$ on three languages than when training only on these languages $(1.03 \%)$. Third, the one-to-many training strategy (efsraz-all, $0.92 \%$ ) performs better than 1:1 (efsra-z, 1.01\%). In addition, it allows to obtain a sentence embedding for all languages, while the common output language is excluded in the 1:1 strategy.

Finally, we have explored whether deep architectures are needed when using an BLSTM encoder and a max-pooling sentence representation (see Table 2). We found no experimental evidence that stacking several BLSTM layers is useful.

\subsection{Many-to-one training strategies}

In this section, we study two M:1 training strategies, namely $2: 1$ and $3: 1$. Since the number of

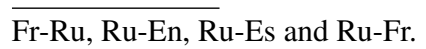




\begin{tabular}{|l||c|c||c|c|c|c|c|c|}
\hline \hline \multirow{2}{*}{ Network } & \multicolumn{3}{c||}{ LSTM + last } & \multicolumn{6}{c|}{ BLSTM + max-pooling } \\
& $3 \times 512$ & $3 \times 1024$ & $1 \times 256$ & $2 \times 256$ & $3 \times 256$ & $1 \times 512$ & $2 \times 512$ & $3 \times 512$ \\
\hline $1: 1$, efsra-Z & 2.51 & - & 1.44 & 1.21 & 1,65 & 1.25 & 1.25 & 1.53 \\
$1:$ M, efsraz-all & 2.38 & 1.89 & 1.27 & 1.30 & 1.53 & $\mathbf{1 . 1 5}$ & 1.17 & 1.30 \\
\hline
\end{tabular}

Table 2: Error rates of similarity search on the UN Dev corpus for five language pairs (efsra). Comparision of LSTMs and BLSTMs of different size and depth.

combinations quickly increases with the number of input languages, we limit these experiences to three input languages (system efs- $a$ ). In that case, we have three 1:1 training paths $(\mathrm{En} \rightarrow \mathrm{Ar}, \mathrm{Fr} \rightarrow \mathrm{Ar}$ and $\mathrm{Es} \rightarrow \mathrm{Ar})$, three 2:1 training paths $(\mathrm{En}+\mathrm{Fr} \rightarrow \mathrm{Ar}$, $\mathrm{En}+\mathrm{Es} \rightarrow \mathrm{Ar}$ and $\mathrm{Fr}+\mathrm{Es} \rightarrow \mathrm{Ar}$ ) and one 3:1 configuration $(\mathrm{En}+\mathrm{Fr}+\mathrm{Es} \rightarrow \mathrm{Ar})$. This is illustrated in Figure 2. To obtain efficient training, we use homogeneous mini-batches, ie. the number of encoders and decoders is constant. Examples in a minibatch are sampled according to a coefficient. In order to make a fair comparison, these resampling coefficient were chosen so that each encoders always sees the same number of sentences (roughly 8.3M). We refer to the different runs with an ID (first column in Table 3). As an example, for the experiment with ID $12_{a}, 90 \%$ of the mini-batches are $1: 1$ and $5 \%$ are $2: 1$. Note that that the $2: 1$ samples have a coefficient of 0.05 since two encoders are simultaneously used.

The first striking result is that presenting all in-

\begin{tabular}{|c|c|c|c|c|}
\hline \multirow[b]{2}{*}{ ID } & \multicolumn{3}{|c|}{ \# input languages } & \multirow{2}{*}{$\begin{array}{c}\text { Similarity } \\
\text { Error }\end{array}$} \\
\hline & 1 & 2 & 3 & \\
\hline \multicolumn{5}{|c|}{ One M:1 strategy } \\
\hline 1 & 1 & - & - & $1.03 \%$ \\
\hline 2 & - & 0.5 & - & $1.85 \%$ \\
\hline 3 & - & - & 1 & $67.9 \%$ \\
\hline \multicolumn{5}{|c|}{ Combining 1:1 and 2:1 strategies } \\
\hline $12_{a}$ & 0.9 & 0.05 & - & $1.09 \%$ \\
\hline $12_{b}$ & 0.8 & 0.10 & - & $1.16 \%$ \\
\hline $12_{c}$ & 0.7 & 0.15 & - & $1.15 \%$ \\
\hline $12_{d}$ & 0.6 & 0.20 & - & $1.13 \%$ \\
\hline $12_{e}$ & 0.5 & 0.25 & - & $1.22 \%$ \\
\hline \multicolumn{5}{|c|}{ Combining $1: 1$ and $3: 1$ strategies } \\
\hline 13 & 0.5 & - & 0.5 & $1.38 \%$ \\
\hline \multicolumn{5}{|c|}{ Combining 1:1, 2:1 and 3:1 strategies } \\
\hline $123_{a}$ & 0.33 & 0.16 & 0.33 & $1.39 \%$ \\
\hline $123_{b}$ & 0.25 & 0.25 & 0.25 & $1.40 \%$ \\
\hline
\end{tabular}

Table 3: Different M:1 strategies for three input languages (system efs-a). The baseline with the $1: 1$ strategy is $1.03 \%$ (line with ID 1). put languages at once and averaging the three sentence representations (3:1, ID 3) does not allow to learn joint representations. We are however able to learn joint representations with the 2:1 strategy (ID 2), but the performance is worse than the $1: 1$ baseline (1.85\% versus $1.03 \%)$. We are also tried to alternate between 1:1 and 2:1 mini-batches with increasing resampling coefficients (ID $12_{a}$ to $12_{e}$ ). The idea is that each encoder learns to provide a sentence representation when used alone and when used with another one. However, we observe that adding 2:1 training paths is not useful: the similarity error increases. The same observation holds when adding 3:1 training paths (ID 13 and 123). Overall, we were not able to improve the baseline of $1.03 \%$ similarity error obtained with a simple 1:1 training strategy. Therefore, we did not try the even more complex M:N paths. This failure could be attributed to the fact that we simply average multiple sentence representations. In future research, we will investigate other possibilities, eg. based on correlation like proposed in (Saha et al., 2016; Chandar et al., 2016).

Detailed similarity search error rates for all six languages, including $\mathrm{Zh}$, of our best system are given in Table 4. Overall, the error rates vary only slightly from the average of $1.2 \%$ although the six languages differ significantly with respect to morphology, inflection, word order, etc. In particular, Chinese is handled as well as the other languages. This is in nice contrast to many other NLP application, in particular NMT, for which the performances on Chinese are significantly below those of other languages. All error rates are below 1.7\%.

\subsection{Large scale out-of domain similarity search}

In this section, we evaluate our sentence representation on out-of domain data. We are not aware of another huge corpus which is 6-way parallel for the same languages than the UN corpus. Therefore, we have selected the Europarl corpus and limit our study to three common languages (En, 


\begin{tabular}{|c||c|c|c|c|c|c||c|}
\hline \multicolumn{1}{|c||}{} & \multicolumn{7}{c|}{ Target language } \\
Src & En & Fr & Es & Ru & Ar & Zh & All \\
\hline En & - & 1.10 & 0.70 & 1.07 & 1.05 & 1.15 & 1.02 \\
Fr & 0.97 & - & 0.95 & 1.55 & 1.65 & 1.68 & 1.36 \\
Es & 0.68 & 1.10 & - & 1.20 & 1.35 & 1.27 & 1.12 \\
Ru & 0.78 & 1.52 & 1.23 & - & 1.32 & 1.32 & 1.23 \\
Ar & 0.78 & 1.52 & 1.07 & 1.48 & - & 1.23 & 1.22 \\
Zh & 0.97 & 1.55 & 1.12 & 1.35 & 1.30 & - & 1.26 \\
\hline All & 0.83 & 1.36 & 1.02 & 1.33 & 1.33 & 1.33 & $\mathbf{1 . 2 0}$ \\
\hline
\end{tabular}

Table 4: Pair-wise error rates of similarity search for 6 languages (UN Dev). Training was performed with a one layer BLSTM with 512 hiddens, max-pooling and the "efsraz-all" strategy.

Fr and Es). After excluding duplicates and limiting the sentence length to fifty tokens, we dispose of almost 1.5 million 3-way parallel sentences.

The two training strategies "efsra-z" and "efsraz-all" achieve the same similarity error rate of about $7.7 \%$. We argue that this is an interesting result given the size of the corpus $(1.46 \mathrm{M}$ sentences) and the fact that it contains several sentences which are very similar (e.g. "The session resumes on DATE"). Using the last state of an LSTM $3 \times 512$ achieves an error rate of $12.2 \%$. Evaluating the similarity error requires the calculation of $1.46 \mathrm{M}^{2}$ distances for each language pair. This can be very efficiently performed with the FAISS open-source toolkit (Johnson et al., 2017) which offers many options to increase the speed of nearest neighbor search. Its implementation of brute-force L2 search was sufficient for our purposes.

\subsection{Examples of multilingual search}

On the next page, we give several examples of similarity search. For each example, we give the query and the five closest sentences. Remember that we use the cosine distance, i.e. the value of 1.0 is a perfect match and smaller values are worse.

The first example in Table 5 shows two simple query sentences for which four paraphrases were found in the Europarl corpus. A more complicated query sentence is used in the second example (see Table 6). For such longer sentences, it is unlikely to find several perfect paraphrases in the indexed corpus. However, the system was able to retrieve sentences which share a lot of the meaning of the query: all cover the topic "punishment of (sexual) crimes, independently of the country the crime is committed in". Finally, examples of cross-lingual similarity search are given in Tables 7 and 8 . In the first example, all five nearest French and Spanish sentences have very similar cosine distances, and all are indeed semantically related. Note that the closest French sentence is not the reference translation, but it nevertheless covers well the topic (its English translation is "I should like to make one remark, however, in response to some of the opinions you have expressed").

Table 8 gives an example where not all retrieved sentences have similar cosine distances. The closest sentence is the correct translation, for French and for Spanish. Both second closest sentences are well related to the query and also have a cosine distance close to the best scoring sentence. The third and following sentences are less related with the query, which is clearly reflected in the substantially lower cosine distance. It's interesting to note that the three closest sentences are all identical, independently of the language. This can be seen as experimental evidence of the quality of the multilingual sentence embeddings.

\section{Conclusion}

We have shown that the framework of NMT with multiple encoders/decoders can be used to learn joint fixed-size sentence representations which exhibit interesting linguistic characteristics. We have explored several training paradigms which correspond to partial paths in the whole architecture. We have proposed a new evaluation protocol of multilingual similarity search which easily scales to many languages and large corpora. We were able to obtain an average cross-lingual similarity error rate of $1.2 \%$ for all 21 languages pairs between six languages ${ }^{5}$ which differ significantly with respect to morphology, inflection, word order, etc. We have also studied the evolution of the similarity error rate when scaling up to 1.4 million sentences, drawn from an out-of-domain corpus.

\section{Acknowledgments}

We would like to thank Ke Tran (Informatics Institute University of Amsterdam, m.k.tran@uva.nl) and Orhan Firat (Middle East Technical University, orhan.firat@ceng.metu.edu.tr, now at Google) for their help with implementing some of the algorithms during their internship at Facebook AI Research in 2016.

\footnotetext{
${ }^{5}$ English, French, Spanish, Russian, Arabic and Chinese.
} 


\begin{tabular}{|ll|ll|}
\hline Query: & All kinds of obstacles must be eliminated. & Query: & I did not find out why. \\
\hline$D_{2}=0.970245$ & All kinds of barriers have to be removed. & $D_{2}=0.913365$ & I have no idea why. \\
$D_{3}=0.799097$ & All these things must be stopped. & $D_{3}=0.913244$ & I fail to see the connection. \\
$D_{4}=0.794444$ & All forms of provocation must be avoided. & $D_{4}=0.906929$ & I do not understand why. \\
$D_{5}=0.792740$ & All forms of violence must be prohibited. & & \\
\hline
\end{tabular}

Table 5: Five closest sentences found by monolingual similarity search in English. All are some form of para-phrasing. The closest sentence (distance $=1$ ) is always identical to the query and therefore omitted.

\begin{tabular}{|ll|}
\hline Query & $\begin{array}{l}\text { All citizens who commit sexual crimes against children must be punished, regardless of whether the } \\
\text { crime is committed within or outside the EU. }\end{array}$ \\
\hline$D_{2}=0.650070$ & $\begin{array}{l}\text { All kinds of sexual abuse of children are criminal and must be seen as the crimes that they are in all Member } \\
\text { States. }\end{array}$ \\
$D_{3}=0.580904$ & $\begin{array}{l}\text { The perpetration of violence against women is a criminal act, whether in public or in private. } \\
D_{4}=0.565544\end{array}$ \\
$D_{5}=0.560186$ & $\begin{array}{l}\text { The perpetrators of crimes cannot be allowed to believe that they will enjoy impunity, regardless of where } \\
\text { they may reside, be it in Europe, in Africa or in any other part of the world. } \\
\text { gardless of their citizenship must be ended. }\end{array}$ \\
\hline
\end{tabular}

Table 6: A more complicated English sentence and the five closest sentences (excluding itself). All cover the punishment of (sexual) crimes.

\begin{tabular}{|c|c|c|}
\hline $\mathbf{E N}_{59104}$ & Query & Allow me, however, to comment on certain issues raised by the honourable Members. \\
\hline $\mathrm{FR}_{390378}$ & $D_{1}=0.678347$ & Je voudrais toutefois apporter un commentaire à quelques-uns de vos avis. \\
\hline $\mathrm{FR}_{59104}$ & $D_{2}=0.676565$ & Permettez-moi toutefois de commenter certaines questions soulevées par les députés. \\
\hline $\mathrm{FR}_{431699}$ & $D_{3}=0.665086$ & $\begin{array}{l}\text { Je voudrais toutefois formuler un certain nombre de commentaires concernant les remarques de } \\
\text { M. Watson. }\end{array}$ \\
\hline $\mathrm{FR}_{651418}$ & $D_{4}=0.660149$ & $\begin{array}{l}\text { Je voudrais toutefois faire part de quelques remarques qui cadrent très bien avec ce que vous } \\
\text { avez évoqué. }\end{array}$ \\
\hline $\mathrm{FR}_{269297}$ & $D_{5}=0.647646$ & $\begin{array}{l}\text { Je voudrais toutefois m'attarder sur certaines recommandations concrètes qui sont adressées à } \\
\text { la Commission. }\end{array}$ \\
\hline $\mathrm{ES}_{59104}$ & $D_{1}=0.693376$ & No obstante, permítanme comentar ciertas cuestiones planteadas por sus Señorías. \\
\hline $\mathrm{ES}_{390378}$ & $D_{2}=0.663397$ & $\begin{array}{l}\text { Sin embargo, quisiera añadir algunas observaciones en relación con algunas de las opiniones } \\
\text { que han manifestado. }\end{array}$ \\
\hline $\mathrm{ES}_{253861}$ & $D_{3}=0.648316$ & Dicho esto, permítanme contestar algunos de los asuntos específicos que ustedes han planteado. \\
\hline $\mathrm{ES}_{133167}$ & $D_{4}=0.637314$ & Permítanme, no obstante, señalar algunas consideraciones que acaban de exponerse. \\
\hline $\mathrm{ES}_{652101}$ & $D_{5}=0.636661$ & No obstante, permítanme que conteste a algunos comentarios que se han realizado. \\
\hline
\end{tabular}

Table 7: Cross-lingual similarity search. English query and the five closest French and Spanish sentences. We also provide the index of the sentences (reference=59104). All the cosine distances are close and the sentences are indeed semantically related.

\begin{tabular}{|lll|}
\hline $\mathbf{E N}_{77777}$ & Query & $\begin{array}{l}\text { And yet the report on the fight against racism does not demonstrate that the necessary } \\
\text { conclusions have been drawn. }\end{array}$ \\
\hline $\mathrm{FR}_{77777}$ & $\mathrm{D}=0.766306$ & $\begin{array}{l}\text { Pourtant, le rapport sur la lutte contre le racisme n'indique pas que l'on en ait tiré les conclusions } \\
\text { qui s'imposent. }\end{array}$ \\
$\mathrm{FR}_{1081193}$ & $\mathrm{D}=0.719267$ & $\begin{array}{l}\text { Ainsi, le rapport sur la lutte contre le racisme n'indique pas que l'on en a tiré les conclusions } \\
\text { qui s'imposent. } \\
\text { Le rapport sur les femmes et le fondamentalisme n'offre toutefois aucune solution à cette } \\
\text { problématique. }\end{array}$ \\
\hline $\mathrm{FR}_{282752}$ & $\mathrm{D}=0.483043$ & $\begin{array}{l}\text { Sin embargo, el informe sobre la lucha contra el racismo no muestra que se hayan extraído las } \\
\text { conclusiones necesarias. } \\
\text { Así, el informe sobre la lucha contra el racismo no muestra que se hayan extraído las conclu- } \\
\text { siones necesarias. } \\
\text { No obstante, el informe acerca de las mujeres y el fundamentalismo no ofrece ninguna solución } \\
\text { para este problema. }\end{array}$ \\
\hline $\mathrm{ES}_{282752}$ & $\mathrm{D}=0.78192193=0.474343$ & $\mathrm{D}=0.735487$ \\
\hline
\end{tabular}

Table 8: Cross-lingual similarity search. English query and the three closest French and Spanish sentences. In both cases, the correct translation was retrieved. The second closest sentences are also semantically well related to the query. However, the third (and following sentences) only cover some of the aspects of the query. This is indeed reflected in the lower similarity score. 


\section{References}

Eneko Agirre, Carmen Banea, Daniel Cer, Mona Diab, Aitor Gonzalez-Agirre, Rada Mihalcea, German Rigau, and Janyce Wiebe. 2016. Semeval-2016 task 1: Semantic textual similarity, monolingual and cross-lingual evaluation. In SemEval workshop.

Sanjeev Arora, Yingyu Liang, and Tengyu Ma. 2017. A simple but tough-to-beat baseline for sentence embeddings. In ICLR.

Dzmitry Bahdanau, Kyunghyun Cho, and Yoshua Bengio. 2015. Neural machine translation by jointly learning to align and translate. In ICLR.

Mauro Cettolo, Christian Girardi, and Marcello Federico. 2012. $\mathrm{Wit}^{3}$ : Web inventory of transcribed and translated talks. In EAMT. pages 261-268.

Sarath Chandar, Mitesh M. Khapra, Hugo Larochelle, and Balaraman Ravindran. 2016. Correlation neural networks. Neural Computation 28:257-285.

Sarath Chandar, Mitesh M. Khapra, Balaraman Ravindran, Vikas Raykar, and Amrita Saha. 2013. Multilingual deep learning. In NIPS DL wshop.

Kyunghyun Cho, Bart van Merrienboer, Caglar Gulcehre, Fethi Bougares, Holger Schwenk, and Yoshua Bengio. 2014. Learning phrase representations using rnn encoder-decoder for statistical machine translation. In EMNLP.

Ronan Collobert and Jason Weston. 2008. A unified architecture for natural language processing: deep neural networks with multitask learning. In ICML. pages $160-167$.

Alexis Conneau, Douwe Kiela, Holger Schwenk, Loic Barrault, and Antoine Bordes. 2017. Supervised learning of universal sentence representations from natural language inference data. In https:// arxiv.org/abs/1705.02364.

J. Coulmance, J.M. Marty, G. Wenzek, and A. Benhaloum. 2015. Trans-gram, fast cross-lingual word embeddings. In EMNLP.

Daxiang Dong, Huan Wu, Wei He, Dianhai Yu, and Haifeng wang. 2015. Multi-task learning for multiple language translation. In $A C L$. pages 1723-1732.

Orhan Firat, Kyunghyun Choa, and Yoshua Bengio. 2016a. Multi-way, multilingual neural machine translation with shared attention mechanism. In NAACL.

Orhan Firat, Baskaran Sankaran, Yaser Al-Onaizan, Fatos T. Yarman Vural, and Kyunghyun Cho. 2016b. Zero-resource translation with multi-lingual neural machine translation. In EMNLP.

Andrea Frome, Grep S. Corrado, Jonathon Shlens, Samy Bengio, Jeffrey Dean, marc' Aurelio Ranzato, and Thomas Mikolov. 2013. DeViSa:E a deep visual-semantic embedding model. In NIPS.
S. Gouews, Y. Bengio, and G. Corrado. 2014. Bilbowa: Fast bilingual distributed representations without word alignments. In ICML.

Karl Moritz Hermann and Phil Blunsom. 2014. Multilingual models for compositional distributed semantics. In ACL. pages 58-68.

Jeff Johnson, Matthijs Douze, and Hervé Jégou. 2017. Billion-scale similarity search with gpus. arXiv preprint arXiv:1702.08734.

Melvin Johnson et al. 2016. Google's multilingual neural machine translation system: Enabling zero-shot translation. In https: / / arxiv.org/ abs/1611.04558.

Nal Kalchbrenner and Phil Blunsom. 2013. Recurrent continuous translation models. In EMNLP. pages 1700-1709.

A. Klementiev, I. Titov, and B. Bhattarai. 2012. Inducing crosslingual distributed representations of words. In Coling.

T. Kociský, K.M. Hermann, and P. Blunsom. 2014. Learning bilingual word representations by marginalizing alignments. In ACL. pages 224-229.

Zhouhan Lin, Minwei Feng, Cicero Nogueira dos Santos, mo Yu, Bing Xiang, Bowen Zhou, and Yoshua Bengio. 2017. A structured self-attentive sentence embedding. In ICLR.

Yang Liu, Chenjie Sun, Lei Lin, and Xiaolong Wang. 2016. Learning natural language inference using bidirectional 1stm model and inner-attention. In https://arxiv.org/abs/1605.09090.

Minh-Thang Luong, Quoc V. Le, Ilya Sutskever, Oriol Vinyals, and Lukasz Kaiser. 2015a. Multi-task sequence to sequence learning. In ICLR.

T. Luong, H. Pham, and C.D. Manning. 2015b. Bilingual word representations with monolingual quality in mind. In ACL workshop on Vector Space Modeling for NLP. pages 151-159.

Tomas Mikolov, Wen tau Yih, and Geoffrey Zweig. 2013. Linguistic regularities in continuous word space representations. In NAACL. pages 746-751.

Aditua Mogadala and Achim Rettinger. 2016. Bilingual word embeddings fomr parallel and nonparallel corpora for cross-language classification. In NAACL. pages 692-702.

Hideki Nakayama and Noriki Nishida. 2016. Zeroresource machine translation by multimodal encoder-decoder network with multimedia pivot. In https://arxiv.org/abs/1611.04503.

Jiquan Ngiam, Aditya Khosla, Mingyu Kim, Juhan Nam, Honglak Lee, and Andrew Y. Ng. 2011. Multimodal deep learning. In ICML. 
Hieu Pham, Minh-Thang Luong, and Christopher D. Manning. 2015. Learning distributed representations for multilingual text sequences. In Workshop on Vector Space Modeling for NLP.

Amrita Saha, Mitesh M. Kharpa, Sarath Chandar, Janarthanan Rajendran, and Kyunghyun Cho. 2016. A correlational encoder decoder architecture for pivot based sequence generation. In https: / / arxiv. org/abs/1606.04754.

Rico Sennrich, Barry Haddow, and Alexandra Birch. 2016. Neural machine translation of rare words with subword units. In $A C L$. pages 1715-1725.

Richard Socher, Jeffrey Pennington, Eric H. Huang, Andrew Y. Ng, and Christopher D. Manning. 2011. Semi-supervised recursive autoencoders for predicting sentiment distributions. In EMNLP.

Ilya Sutskever, Oriol Vinyals, and Quoc V. Le. 2014. Sequence to sequence learning with neural networks. In NIPS. pages 3104-3112.

Yonghui Wu et al. 2016. Google's neural machine translation system: Bridging the gap between human and machine translation. In https://arxiv. org/abs/1610.05011.

Xiang Zhang, Junbo Zhao, and Yann LeCun. 2015. Character-level convolutional networks for text classification. In NIPS. pages 649-657.

Han Zhao, Zhengdong Lu, and Pascal Poupart. 2015. Self-adaptive hierarchical sentence model. In https://arxiv.org/abs/1504.05070.

Jie Zhou, Ying Cao, Xuguang Wang, Peng Li, and Wei Xu. 2016a. Deep recurrent models with fast-forward connections for neural machine translation. TACL $4: 371-383$

Xinjie Zhou, Xiaojun Wan, and Jianguo Xiao. 2016b. Cross-lingual sentiment classification with bilingual document representation learning. In $A C L$.

M Ziemski, Marcin Juncys-Dowmunt, and B. Pouliquen. 2016. The united nations parallel corpus v1.0. In $L R E C$.

Barret Zoph and Kevin Knight. 2016. Multi-source neural translation. In $N A A C L$. pages 30-34. 